\title{
Clinical implications of chimerism after allogeneic hematopoietic stem cell transplantation in children with non-malignant diseases
}

\author{
Meerim Park ${ }^{1}$, Kyung Nam $\mathrm{Koh}^{2}$, Jong Jin Seo ${ }^{2}$, Ho Joon $\mathrm{Im}^{2}$ \\ Department of Pediatrics, ${ }^{1}$ College of Medicine, Chungbuk National University, Cheongju, ${ }^{2}$ Asan Medical Center Children's Hospital, \\ University of Ulsan College of Medicine, Seoul, Korea
}

p-ISSN 1738-7949 / e-ISSN 2092-9129

http://dx.doi.org/10.5045/kjh.2011.46.4.258

Korean J Hematol 2011;46:258-64.

Received on November 30, 2011

Revised on December 5, 2011

Accepted on December 7, 2011

\author{
Correspondence to \\ Ho Joon Im, M.D., Ph.D. \\ Department of Pediatrics, Asan Medical \\ Center Children's Hospital, University of \\ Ulsan College of Medicine, Pungnap-dong, \\ Songpa-gu, Seoul 138-736, Korea \\ Tel: +82-2-3010-3371 \\ Fax: $+82-2-473-3725$ \\ E-mail: hojim@amc.seoul.kr \\ (C) 2011 Korean Society of Hematology
}

\section{Background}

The effects of chimerism on outcomes following allogeneic hematopoietic stem cell transplantation (HSCT) are unclear and may differ between diseases. We retrospectively evaluated the association between chimerism and transplant outcomes in children with nonmalignant diseases.

\begin{abstract}
Methods
Chimerism was evaluated using short-tandem repeat polymerase chain reaction (STR-PCR) in 48 patients, with mixed chimerism (MC) defined as greater than $1 \%$ recipient cells.

Results

The only variable exerting a significant influence on patients' chimerism status was the number of infused CD34 + cells. MC was detected in 23 transplants (9 showing transient MC; 10 with sustained low levels [ $\leq 30 \%$ ] of autologous cells; and 4 with high-level MC [ $>30 \%]$ ). The degree of STR-PCR at 28 days after HSCT was significantly higher in patients with high-level MC than those with transient or low-level MC. All patients with transient or low-level MC successfully maintained engraftment and showed a clinical response to $\mathrm{HSCT}$, whereas 2 of the 4 patients with high-level MC experienced graft failure. The incidences of grades II-IV acute and chronic graft-versus-host disease (GVHD) were significantly higher in patients with complete donor chimerism (CC) than MC. We observed no significant survival differences between $\mathrm{CC}$ and $\mathrm{MC}$ groups. However, the survival rate was lower in patients with high $M C$ than those with low-level or transient $M C$ $(P=0.03)$.
\end{abstract}

\section{Conclusion}

In non-malignant diseases, MC may indicate a tolerant state with a decreased incidence of GVHD. However, high-level MC may signify an increased risk of graft failure and a lower survival rate.

Key Words Non-malignant disease, Allogeneic hematopoietic stem cell transplantation, Chimerism

\section{INTRODUCTION}

Allogeneic hematopoietic stem cell transplantation (Allo-HSCT) is a well-established treatment for several nonmalignant diseases, including severe aplastic anemia (SAA), inherited bone marrow failure (BMF) syndromes, immunodeficiencies, and metabolic disorders [1]. Although complete donor hematopoiesis is a desirable outcome of allo-HSCT in malignant disorders, complete replacement of the recipient's hematopoietic system is not considered necessary to improve the underlying disease state in patients with non-malignant disorders. This coexistence of host and donor hematopoietic cells is known as mixed chimerism (MC).

Rates of graft failure and rejection are high in patients with non-malignant diseases [2, 3]. MC has been linked to graft rejection $[4,5]$, suggesting that early testing for posttransplant chimerism may help to identify patients at increased risk of graft rejection, who may therefore require immunotherapy [6]. Although patients with a significant

\footnotetext{
This is an Open Access article distributed under the terms of the Creative Commons Attribution Non-Commercial License (http://creativecommons.org/licenses/by-nc/3.0)
} which permits unrestricted non-commercial use, distribution, and reproduction in any medium, provided the original work is properly cited. 
proportion of host cells are known to be at increased risk of rejection in cases of malignant disease, only a few studies have focused exclusively on children with non-malignant diseases [7, 8]. Furthermore, the utility of chimerism in predicting subsequent transplant outcomes in non-malignant diseases has not yet been established.

The main aims of this study were to identify the variables affecting chimerism and to determine the impact of MC on transplantation outcomes in patients undergoing allo-HSCT for non-malignant diseases.

\section{MATERIALS AND METHODS}

\section{Patients and donors}

Between April 2000 and March 2011, 48 children underwent 50 allo-HSCTs for non-malignant diseases at the Asan Medical Center Children's Hospital in Seoul, Korea. In this study, we analyzed the initial HSCT data from all patients. Median age at first HSCT was 8.4 years (range, 0.6-20.5 years), and the median duration of follow-up was 41 months (range, 8-138 months). Diseases were classified as BMF or non-BMF. All donor-recipient pairs were fully typed for HLA-A, -B, -C, and -DR using high-resolution molecular typing. As a surrogate marker of iron overload at the time of transplantation, serum ferritin was routinely measured as a part of the pretransplant work-up before the beginning of the conditioning regimen using a 2-site sandwich immunoassay with direct chemiluminescence (ADVIA Centaur, Siemens). All patients were stable, without fever or other events, suggesting that pretransplant ferritin levels are a good estimator of iron status and are not significantly affected by an inflammatory state. Data were analyzed in October 2011. The demographic and clinical characteristics of patients and donors are summarized in Table 1 . This retrospective study was approved by the institutional review board at the Asan Medical Center in Seoul, Korea.

\section{Graft-versus-host disease}

Acute and chronic graft-versus-host diseases (GVHD) were graded according to the established criteria [9].

\section{Analysis of chimerism}

Whole blood samples were collected from individual donors and recipients at 1 (day 28), 2, 3, 6, and 12 months after allo-HSCT. In cases where MC was detected, chimerism was tested at more frequent intervals. DNA was prepared from whole blood samples using the QIAamp Blood Kit (Qiagen, Hilden, Germany) and quantified spectrophotometrically. Polymorphic short-tandem repeat (STR) markers were amplified using the AmpFlSTR Profiled Plus PCR amplification kit (Applied Biosystems, Foster City, CA, USA). Amplified fragments were analyzed using an ABI PRISM 310 Genetic Analyzer (Applied Biosystems), and peak areas were quantified using Genescan software (Applied Biosystems). The percentages of donor and recipient DNA were calculated from individual proportions of donor and recipient
Table 1. Characteristics of patients and donors.

\begin{tabular}{|c|c|}
\hline & $\mathrm{N}$ \\
\hline Patients & 48 \\
\hline Follow-up duration, months, median (range) & $41(8-138)$ \\
\hline Male/Female & $36 / 12$ \\
\hline Median age, years, median (range) & $8.4(0.6-20.5)$ \\
\hline No. CD34 $\times 10^{6} / \mathrm{kg}$ infused, median (range) & $8.38(1.0-32.9)$ \\
\hline \multicolumn{2}{|l|}{ Diseases } \\
\hline Bone marrow failure (BMF) & 37 \\
\hline Severe aplastic anemia & 27 \\
\hline Fanconi anemia & 4 \\
\hline Other diseases in $\mathrm{BMF}^{\mathrm{a}}$ ) & 6 \\
\hline Hemophagocytic lymphohistiocytosis & 5 \\
\hline Chronic granulomatous disease & 1 \\
\hline Wiskott-Aldrich syndrome & 1 \\
\hline Severe combined immunodeficiency & 1 \\
\hline Neurodegenerative diseases $\mathrm{s}^{\mathrm{b})}$ & 3 \\
\hline \multicolumn{2}{|l|}{ Donors } \\
\hline Matched related (8/8) & 11 \\
\hline \multicolumn{2}{|l|}{ Mismatched related } \\
\hline 1 mismatch $(7 / 8)$ & 2 \\
\hline 2 mismatches $(6 / 8)$ & 1 \\
\hline$\geq 3$ mismatches $(\leq 5 / 8)$ & 8 \\
\hline Matched unrelated (8/8) & 15 \\
\hline \multicolumn{2}{|l|}{ Mismatched unrelated } \\
\hline 1 mismatch $(7 / 8)$ & 7 \\
\hline 2 mismatches $(6 / 8)$ & 3 \\
\hline$\geq 3$ mismatches $(\leq 5 / 8)$ & 1 \\
\hline \multicolumn{2}{|l|}{ Stem cell source } \\
\hline Bone marrow & 17 \\
\hline Peripheral blood & 27 \\
\hline Cord blood & 4 \\
\hline \multicolumn{2}{|l|}{ Conditioning regimen } \\
\hline \multicolumn{2}{|l|}{ BMF } \\
\hline Myeloablative & 8 \\
\hline Reduced & 29 \\
\hline \multicolumn{2}{|l|}{ Non-BMF } \\
\hline Myeloablative & 7 \\
\hline Reduced & 4 \\
\hline \multicolumn{2}{|l|}{ T-cell depletion } \\
\hline Yes & 9 \\
\hline No & 39 \\
\hline
\end{tabular}

${ }^{a)}$ Other diseases in BMF: 2 pure red cell anemia, 2 congenital amegakaryocytic thrombocytopenia, 1 Kostmann disease, and 1 congenital dyserythropoietic anemia. ${ }^{\text {b) Neurodegenerative disea- }}$ ses: 2 Krabbe disease and 1 adrenoleukodystrophy.

peak areas [10]. Unless otherwise stated, the degrees of STR-PCR and MC were based on host portions.

\section{Definition of chimerism and responses}

Patients were stratified individually based on serial STR-PCR analyses, with chimerism defined as described previously $[7,8]$. Patients with samples showing $1 \%$ or less autologous signals after HSCT were classified as having achieved complete donor chimerism (CC). We selected a cutoff of $1 \%$, since microsatellite and several tandem repeat methods have a sensitivity limit of $1-5 \%$. MC was defined as having a recipient fraction of $1 \%$ to $99 \%$ at any time after HSCT. Patients initially classified as having MC who turned into CC were classified in the transient MC group, while those showing sustained autologous signals greater than $1 \%$ 
were categorized in the sustained MC group. In the sustained MC group, patients consistently displaying autologous signals that did not exceed $30 \%$ were considered to have low-level MC, while those displaying a significant increase in autologous cells, up to $30 \%$, were considered to have high-level MC. Patients with more than $99 \%$ recipient signals were defined as having attained complete recipient chimerism (RC) or autologous reconstitution.

Since there is no clear consensus on the definition of clinical remission in patients with non-malignant diseases, we assessed the response relative to correction of the underlying disease using disease-specific measures. These included normal hemograms along with transfusion independence in patients with BMF syndromes, immune reconstitution and lack of infection in patients with immunodeficiencies, enzyme concentrations and the results of brain imaging and neurologic examinations in patients with metabolic disorders, and absence of symptoms and discontinuation of pretransplant-specific therapies in patients with autoimmune disorders [11]. A complete response (CR) was defined as a normal hemogram with eradication of the underlying disease. A partial response (PR) was defined as the presence of some positive effects of HSCT, but not fulfillment of the criteria for $\mathrm{CR}$ (e.g., patients with BMF that displayed some improvement, but were still transfusion dependent). Disease progression was defined as the absence of positive effects of HSCT.

Graft failure comprises 2 clinical entities: (1) failure to achieve an absolute neutrophil count of $0.5 \times 10^{9} / \mathrm{L}$ or less and marrow hypoplasia for less than 60 days with or without the existence of donor-type hematopoiesis, and (2) complete loss of donor-type hematopoiesis occurring anytime after transplantation. Late graft failure was defined as the loss of the graft after engraftment during follow-up.

\section{Statistics}

The probabilities of overall survival (OS) and event-free survival (EFS) were estimated using the Kaplan-Meier method. An event was defined as graft failure or death either due to treatment toxicity or disease progression. Fisher's exact test was used to compare differences in categorical variables affecting chimerism and response to HSCT between groups, whereas the Mann-Whitney $U$-test was used to compare continuous variables. We also attempted to model relationships between 2 continuous variables by linear regression. For multivariate analysis, the logistic regression model was used. Rreceiver operating characteristic (ROC) plot analysis was performed to determine the threshold value of CD34 + cells for predicting chimerism. Probabilities of acute and chronic GVHD were calculated using the cumulative-incidence-function method; death without GVHD was the competing event. All statistical analyses were performed using the SPSS statistical program (SPSS Inc., Chicago, IL, USA) or R 2.10.1 software (The R Foundation for Statistical Computing, 2007). All $P$-values were 2 -sided, with $P<0.05$ considered statistically significant.

\section{RESULTS}

Forty-eight patients underwent allo-HSCT, and 2 required a second transplant due to graft failure after the first HSCT. At a median follow-up of 41 months, the 3-year OS and EFS rates were $91.5 \pm 4.1 \%$ and $85.3 \pm 5.1 \%$, respectively. Four patients died, 1 due to graft failure and the other 3 due to transplant-related complications, including veno-occlusive disease, hemorrhage, multi-organ failure, and infection.

\section{Chimerism}

During follow-up, 23 patients (48\%) showed sustained CC and $23(48 \%)$ showed MC. Peak MC in the groups of patients with transient $(\mathrm{N}=9)$, low-level $(\mathrm{N}=10)$, and highlevel $(\mathrm{N}=4) \mathrm{MC}$ occurred at a median of 3, 3.5, and 2.3 months, respectively. Patients with transient $\mathrm{MC}$ achieved CC at a median of 7.7 months after HSCT. All 4 patients with high-level MC showed more than $30 \%$ autologous signals within 2 months after HSCT. The chimeric status of patients with high-level MC is shown in Fig. 1.

Two of the 4 patients with high-level MC subsequently experienced graft failure after HSCT (Table 2). One of these patients (UPN 88) achieved CR after the second HSCT, but died due to transplant-related complications resulting in multiple organ failure after 5 months. The second patient (UPN 127) received 2 donor lymphocyte infusions (DLIs), but did not achieve stable engraftment, and subsequently died due to infection and pulmonary hemorrhage.

RC was detected on day 28 after HSCT in 2 patients, 1 with hemophagocytic lymphohistiocytosis (HLH) and 1 with SAA. Although the patient with HLH experienced graft failure, BM showed autologous recovery, and the patient

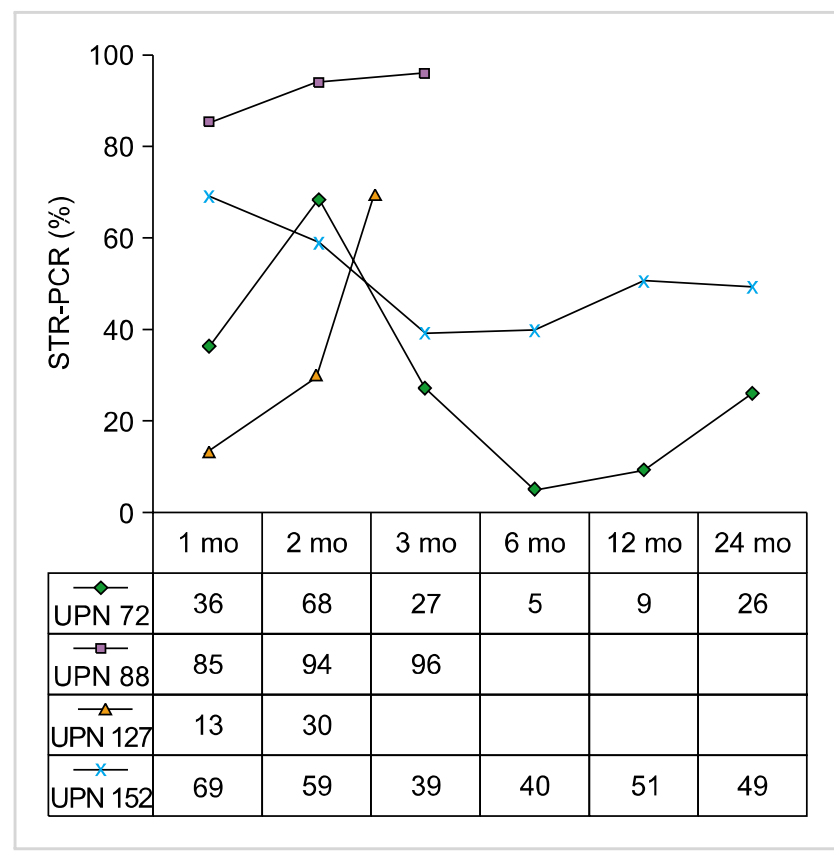

Fig. 1. Chimeric status of patients with high-level mixed chimerism. 
Table 2. Characteristics of patients with high-level mixed chimerism.

\begin{tabular}{|c|c|c|c|c|c|c|c|c|c|c|c|c|}
\hline UPN & Diagnosis & $\begin{array}{l}\text { Age } \\
(\mathrm{yr}) / \\
\text { sex }\end{array}$ & Donor & $\begin{array}{c}\text { Conditioning } \\
\text { regimen }\end{array}$ & TCD & $\begin{array}{c}\text { CD34+ } \\
\text { cells } \\
\left(\times 10^{6} /\right. \\
\mathrm{kg})\end{array}$ & Ferritin & $\begin{array}{l}\text { Peak recipient } \\
\text { chimerism } \\
(\mathrm{mo})\end{array}$ & $\begin{array}{l}\text { Graft failure } \\
\text { (documented } \\
\text { day) }\end{array}$ & aGVHD & $\begin{array}{l}\text { Salvage } \\
\text { strategy }\end{array}$ & $\begin{array}{c}\text { Outcome } \\
\text { (follow-up } \\
\text { months) }\end{array}$ \\
\hline 72 & PRCA & $7.2 / \mathrm{M}$ & MRD & CyATG & - & 40.17 & ND & $68 \%$ (2 mo) & No & No & No & $\mathrm{CR}(+91)$ \\
\hline 88 & SAA & $12.8 / \mathrm{F}$ & MMUD & СУАТG & - & 2 & 256 & $96 \%$ (3 mo) & Yes $(61 \mathrm{~d})$ & No & $2^{\text {nd }} \mathrm{HSCT}^{\mathrm{a})}$ & Death (13) \\
\hline 127 & SAA & $12.9 / \mathrm{M}$ & MMRD & СуATGTBI & + & 4.5 & 5122 & $66 \%(2.5 \mathrm{mo})$ & Yes (61 d) & No & $\mathrm{DLI}^{\mathrm{b})}$ & Death (2) \\
\hline 152 & KD & $4.1 / \mathrm{M}$ & MRD & BuCy & - & 12.09 & 31 & $69 \%$ (1 mo) & No & No & No & $\mathrm{CR}(+53)$ \\
\hline
\end{tabular}

All time variables are presented on the basis of the transplantation date.

a) Patient 88 achieved stable engraftment after the second HSCT, but died of transplant-related complications that resulted in multiple organ failure 5 months after the second HSCT. ${ }^{\text {b) }}$ Although patient 127 received a second DLI, he did not achieve engraftment and died of transplant-related complications 81 days after HSCT.

Abbreviations: PRCA, pure red cell anemia; SAA, severe aplastic anemia; KD, Kostmann disease; MRD, matched related donor; MMUD, mismatched unrelated donor; MMRD, mismatched related donor; $\mathrm{Cy}$, cyclophosphamide; ATG, antithymocyte globulin; TBI, total body irradiation; Bu, busulfan; TCD, T-cell depletion; DLI, donor lymphocyte infusion; CR, complete remission; ND, not done.

remained in CR at data cut-off. The SAA patient achieved neutrophil engraftment on day 11 (10\% autologous portion of MC on day 20), but neutropenia occurred at day 14, and STR-PCR revealed $100 \%$ host signal at day 28 . Owing to graft failure, the patient underwent a second HSCT and achieved CC with stable engraftment.

\section{STR-PCR at day 28 post-HSCT}

We observed a linear relationship between the degree of day-28 STR-PCR and pretransplant ferritin concentrations. Higher ferritin concentrations were significantly associated with a higher recipient proportion of MC by day 28 after HSCT $\left(r^{2}=0.2, P=0.03\right)$. At day 28 after HSCT, STR-PCR values were significantly higher in patients with high-level MC than those with transient or low-level MC (50.8\% vs. $3.7 \%, P<0.01$ ).

We observed a linear relationship between the day-28 STR-PCR and time to engraftment. Specifically, a high proportion of autologous cells at day 28 after HSCT were significantly associated with late neutrophil $\left(r^{2}=0.24, P<0.01\right)$ and platelet $\left(r^{2}=0.15, P=0.02\right)$ engraftment. No differences were evident in the day-28 STR-PCR between patients with PR and CR. However, STR-PCR at day 28 was significantly lower in the response than the non-response group $(6.1 \%$ vs. $66.0 \%, P<0.01)$.

\section{Variables affecting chimerism}

The variables tested for effects on chimerism included patient age, disease category, numbers of infused CD34+ and CD3+ cells, source of stem cells, donor type, degree of HLA match, pretransplant serum ferritin concentration, and intensity of the conditioning regimen. Increasing $\mathrm{MC}$ was only observed in patients with BMF. The number of infused CD34 + cells was significantly higher in the CC group than in the MC group $\left(13.2 \times 10^{6} / \mathrm{kg}\right.$ vs. $7.2 \times 10^{6} / \mathrm{kg}$, respectively; $P<0.01$ ). Similarly, we observed a marked increase in the number of infused CD3 + cells in the CC group compared to the MC group $\left(4.92 \times 10^{8} / \mathrm{kg}\right.$ vs. $2.61 \times$ $10^{8} / \mathrm{kg}$, respectively; $\left.P=0.02\right)$. The only variable with a sig-

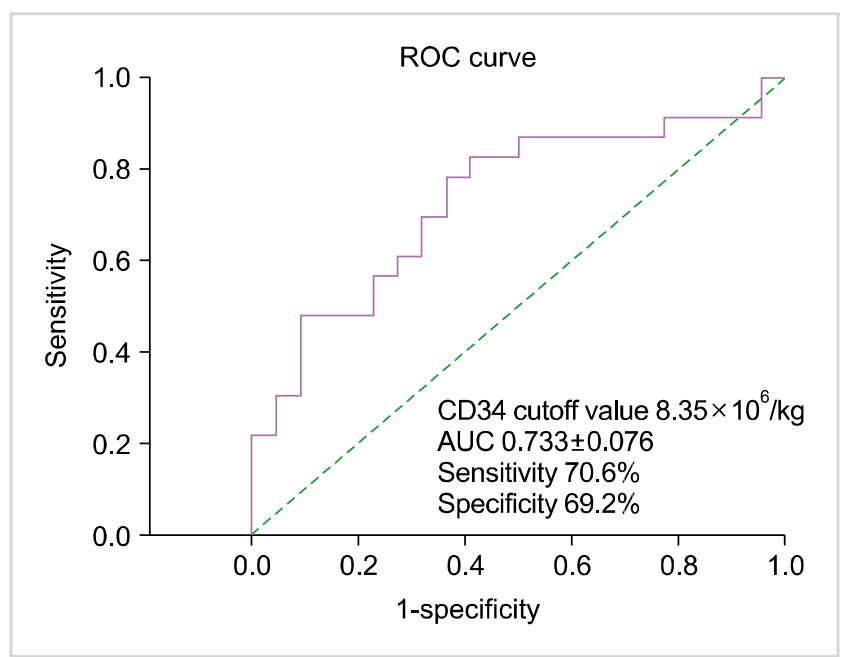

Fig. 2. Receiver operating characteristic curve used to determine the optimal CD34 + cell number cut-off value for predicting chimerism.

nificant influence on patients' chimerism status was the number of infused CD34+ cells, as determined by multivariate analysis. Using ROC curves, the optimal cut-off value of CD34 + cells for predicting the chimerism status was estimated to be $8.35 \times 10^{6} / \mathrm{kg}$ (70.6\% sensitivity, $69.2 \%$ specificity), indicating that patients who received less than $8.35 \times$ $10^{6} / \mathrm{kg}$ of CD34 + cells are at higher risk of developing MC (Fig. 2).

\section{Chimerism and transplantation outcomes}

The relationship between chimerism status and clinical response is shown in Table 3. All patients with CC and low-level/transient MC showed clinical responses to HSCT. Among the 23 patients with posttransplant CC, 20 achieved $\mathrm{CR}$, including 1 who experienced late graft failure 39 months after HSCT, but achieved CR after immunosuppressive therapy. The remaining 3 patients achieved PR, 1 each with adrenoleukodystrophy, Krabbe disease, and SAA. Among the 23 patients with posttransplant MC, 21 showed clinical re- 
Table 3. Posttransplantation outcome according to chimerism status.

\begin{tabular}{|c|c|c|c|c|c|c|}
\hline & \multirow{2}{*}{$\begin{array}{c}\text { Patients } \\
\mathrm{N}=48\end{array}$} & \multirow{2}{*}{$\begin{array}{c}\mathrm{CC} \\
\mathrm{N}=23\end{array}$} & \multicolumn{3}{|c|}{ MC } & \multirow{2}{*}{$\begin{array}{c}\mathrm{RC} \\
\mathrm{N}=2\end{array}$} \\
\hline & & & $\begin{array}{c}\text { Transient } \\
\mathrm{N}=9\end{array}$ & $\begin{array}{c}\text { Low-level } \\
\mathrm{N}=10\end{array}$ & $\begin{array}{l}\text { High-level } \\
\mathrm{N}=4\end{array}$ & \\
\hline CR & 39 & 20 & 8 & 8 & 2 & 1 \\
\hline PR & 6 & 3 & 1 & 2 & 0 & 0 \\
\hline$N R$ & 3 & 0 & 0 & 0 & 2 & 1 \\
\hline Graft failure & 5 & $1^{\text {a) }}$ & 0 & 0 & 2 & 2 \\
\hline Primary & 2 & 0 & 0 & 0 & 1 & 1 \\
\hline Late & 3 & $1^{\text {a) }}$ & 0 & 0 & 1 & 1 \\
\hline $2^{\text {nd }} \mathrm{TPL}$ & 2 & 0 & 0 & 0 & 1 & 1 \\
\hline DLI & 1 & 0 & 0 & 0 & 1 & 0 \\
\hline Death & 4 & $1^{\text {b) }}$ & 0 & $1^{\text {b) }}$ & $1^{\text {b) }}+1^{\text {c) }}$ & 0 \\
\hline
\end{tabular}

a) Although the patient maintained CR with CC for 39 months after transplantation, he showed bone marrow hypoplasia in spite of having CC and was rescued by immunosuppressive therapy. ${ }^{\text {b) }}$ Died of transplant-related complications with partial or complete response to the transplantation. ${ }^{\mathrm{c}}$ Died of graft failure.

Abbreviations: $C C$, complete donor chimerism; $M C$, mixed chimerism; $R C$, recipient chimerism; $C R$, complete response; PR, partial response; $\mathrm{NR}$, no response; TPL, transplantation; DLI, donor lymphocyte infusion.

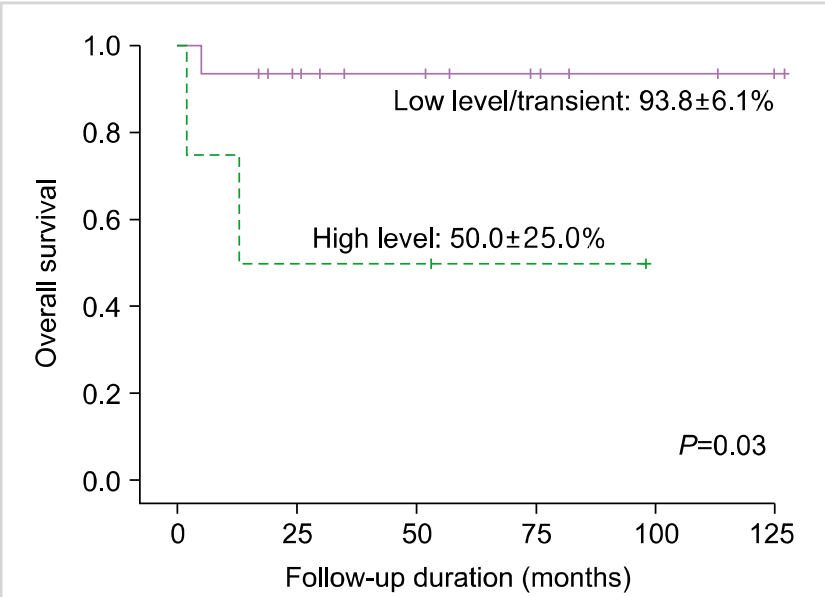

Fig. 3. Kaplan-Meier curves showing overall survival in high-level mixed chimerism and transient or low-level mixed chimerism groups.

sponses to HSCT. Two patients with high-level MC experienced graft failure. The incidence of $\mathrm{CR}$ in the transient, low-level, and high-level MC subgroups was $89 \%(8 / 9), 80 \%$ $(8 / 10)$, and $50 \%(2 / 4)$, respectively $(P=0.03)$. The incidence of graft failure was significantly higher in the high-level MC subgroup than the low-level and transient MC subgroups $(P=0.03)$. Higher ferritin concentrations before HSCT $(P<$ $0.01)$ and lower numbers of infused CD34 + cells $(P=0.01)$ were additionally associated with graft failure.

The incidences of grades II?IV acute and chronic GVHD were significantly higher in CC patients than in MC patients (56.5\% vs. $13.0 \%, P<0.01$; and $47.8 \%$ vs. $21.7 \%, P=0.04$, respectively). No significant differences in survival were evident between the CC and MC groups. However, the OS rate was significantly lower in patients with high-level $\mathrm{MC}$ than in patients with low-level and transient MC (50.0\% vs. $93.8 \%, P=0.03$; Fig. 3). The EFS rate of patients with high-level and low-level/transient MC was $87.5 \%$ and $50.0 \%$,

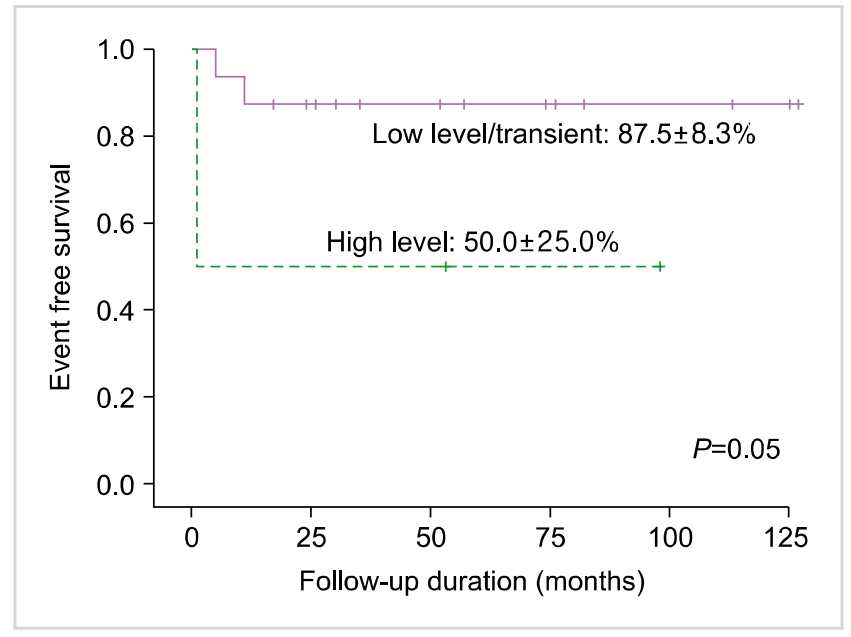

Fig. 4. Kaplan-Meier curves showing event-free survival in high-level mixed chimerism and transient or low-level mixed chimerism groups.

respectively ( $P=0.05$; Fig. 4$)$. The causes of death in the high-level MC group included graft failure and multi-organ failure after second HSCT.

\section{DISCUSSION}

This study presents a retrospective analysis of chimerism status and the effects of chimerism on clinical outcomes in children undergoing HSCT for non-malignant diseases. Allo-HSCT was considered the only curative approach for non-malignant diseases in our patients. In our cohort of 48 patients receiving 50 allo-HSCTs, 5 showed graft failure. The overall survival rate was $91.5 \%$, similar to that reported in earlier studies involving patients with non-malignant diseases $[2,12]$.

Unlike hematologic malignancies, where $100 \%$ donor engraftment is always desired, the number of donor cells re- 
quired for sustained engraftment and correction of underlying disease in patients with non-malignant disorders may be host- and disease-specific. The incidence of MC in our study was $48 \%$, similar to that reported previously [13]. Possible factors associated with MC development include the source of hematopoietic stem cells, intensity of the conditioning regimen, composition of the hematopoietic graft, and type of donor [14-16]. In our experiments, the only variable that had a significant influence on the achievement of MC was the number of infused CD34+ cells, as determined by multivariate analysis. Baron et al. [17] suggested that more rapid reconstitution of the $\mathrm{T}$-cell system and reduced rejection rates could be achieved by increasing the dose of CD34+ cells administered. Similar findings have been reported in other studies $[18,19]$.

While no significant differences were evident in transplant outcomes between patients with MC and those with CC, acute and chronic GVHD rates were lower in the MC group. Furthermore, all patients with low-level or transient MC successfully maintained engraftment and showed clinical responses to HSCT. The lower incidence of GVHD in the MC group may be attributed to a decrease in alloreactive T-cells that attack recipient tissues or an imbalance between pro-inflammatory and immune-modulating cytokines [20].

STR-PCR at day 28 after allo-HSCT had a predictive value for transplantation outcomes. The degree of STR-PCR was significantly lower in responders than nonresponders. Moreover, day-28 STR-PCR facilitated the identification of patients at risk of high-level MC. Therefore, the degree of chimerism at day 28 after allo-HSCT may be used as a novel platform for additional therapy to induce CR.

High-level MC is correlated with an increased risk of graft failure and rejection [21]. Although we observed no significant differences in survival between the CC and MC groups, we found that patients with high-level MC displayed a higher rate of graft failure and lower survival than those with low-level or transient MC. MC is not necessarily associated with graft rejection, but patients with more than $30 \%$ recipient cells in serial samples may be at a higher risk of graft failure. However, no graft loss was observed in 50\% of patients with increasing MC. Thus, the risk of graft rejection after allo-HSCT may be limited to the period before the development of tolerance, suggesting that chimerism testing is not predictive of rejection once tolerance to donor cells has been established. Our understanding of tolerance development following allo-HSCT for non-malignant diseases is still incomplete.

In patients undergoing allo-HSCT with either myeloablative conditioning or reduced intensity conditioning for hematologic malignancies, elevated pretransplant serum ferritin concentration, a surrogate marker of iron overload, has been associated with lower OS and increased nonrelapse mortality [22-24]. We observed an association of pretransplant ferritin concentration with day-28 STR-PCR, but not chimerism status thereafter, during the first year after HSCT. Patients experiencing graft failure displayed extremely high ferritin concentrations. Iron overload is known to be asso- ciated with free radical generation and tissue damage [23]. Overloaded iron may have accumulated in the BM, resulting in deterioration of microenvironmental functions. However, the number of patients with graft failure was relatively small, suggesting the need for larger-scale studies.

Our study had some limitations. First, the retrospective study design, small sample size, and heterogeneous diseases, and transplantation procedures may have precluded meaningful analyses. Nevertheless, this was a relatively large-scale investigation of this rare disease state in children, managed uniformly in a single center. Next, we did not evaluate lineage-specific chimerism in individual patients. Finally, since treatment was based on individual clinical situations, we did not analyze what interventions may have been prompted by the results of chimerism assays, thus affecting patients' clinical outcomes.

In summary, our findings suggest that posttransplant MC in non-malignant diseases indicates a tolerant state associated with decreased incidence of acute and chronic GVHD. MC may thus be sufficient to cure non-malignant disease. However, patients subsequently presenting with more than $30 \%$ recipient cells in serial samples may be at a higher risk of graft failure. The degree of chimerism at day 28 after HSCT may allow the prediction of subsequent clinical responses to HSCT. Our findings collectively highlight the potential value of serial chimerism assays combined with clinical evaluation in providing information on transplantation outcomes.

\section{REFERENCES}

1. Ringden $\mathrm{O}$, Remberger $\mathrm{M}$, Svahn $\mathrm{BM}$, et al. Allogeneic hematopoietic stem cell transplantation for inherited disorders: experience in a single center. Transplantation 2006;81:718-25.

2. Peters C, Steward CG. Hematopoietic cell transplantation for inherited metabolic diseases: an overview of outcomes and practice guidelines. Bone Marrow Transplant 2003;31:229-39.

3. Srinivasan R, Takahashi Y, McCoy JP, et al. Overcoming graft rejection in heavily transfused and allo-immunised patients with bone marrow failure syndromes using fludarabine-based haematopoietic cell transplantation. Br J Haematol 2006;133: 305-14.

4. McCann SR, Bacigalupo A, Gluckman E, et al. Graft rejection and second bone marrow transplants for acquired aplastic anaemia: a report from the Aplastic Anaemia Working Party of the European Bone Marrow Transplant Group. Bone Marrow Transplant 1994; 13:233-7.

5. Bacigalupo A, Brand R, Oneto R, et al. Treatment of acquired severe aplastic anemia: bone marrow transplantation compared with immunosuppressive therapy-The European Group for Blood and Marrow Transplantation experience. Semin Hematol 2000; 37:69-80.

6. Lawler M, McCann SR, Marsh JC, et al. Serial chimerism analyses indicate that mixed haemopoietic chimerism influences the probability of graft rejection and disease recurrence following allogeneic stem cell transplantation (SCT) for severe aplastic 
anaemia (SAA): indication for routine assessment of chimerism post SCT for SAA. Br J Haematol 2009;144:933-45.

7. Hoelle W, Beck JF, Dueckers G, et al. Clinical relevance of serial quantitative analysis of hematopoietic chimerism after allogeneic stem cell transplantation in children for severe aplastic anemia. Bone Marrow Transplant 2004;33:219-23.

8. Willasch A, Hoelle W, Kreyenberg H, et al. Outcome of allogeneic stem cell transplantation in children with non-malignant diseases. Haematologica 2006;91:788-94.

9. Ferrara JL, Deeg HJ. Graft-versus-host disease. N Engl J Med 1991;324:667-74.

10. Nollet F, Billiet J, Selleslag D, Criel A. Standardisation of multiplex fluorescent short tandem repeat analysis for chimerism testing. Bone Marrow Transplant 2001;28:511-8.

11. Svenberg P, Mattsson J, Ringdén O, Uzunel M. Allogeneic hematopoietic SCT in patients with non-malignant diseases, and importance of chimerism. Bone Marrow Transplant 2009;44: 757-63.

12. Boelens JJ, Wynn RF, O'Meara A, et al. Outcomes of hematopoietic stem cell transplantation for Hurler's syndrome in Europe: a risk factor analysis for graft failure. Bone Marrow Transplant 2007;40:225-33.

13. Ozyurek E, Cowan MJ, Koerper MA, Baxter-Lowe LA, Dvorak CC, Horn BN. Increasing mixed chimerism and the risk of graft loss in children undergoing allogeneic hematopoietic stem cell transplantation for non-malignant disorders. Bone Marrow Transplant 2008;42:83-91.

14. Roy DC, Tantravahi R, Murray C, et al. Natural history of mixed chimerism after bone marrow transplantation with CD6-depleted allogeneic marrow: a stable equilibrium. Blood 1990;75:296-304.

15. Baron F, Little MT, Storb R. Kinetics of engraftment following allogeneic hematopoietic cell transplantation with reducedintensity or nonmyeloablative conditioning. Blood Rev 2005;19: 153-64.

16. Sugita J, Tanaka J, Hashimoto A, et al. Influence of conditioning regimens and stem cell sources on donor-type chimerism early after stem cell transplantation. Ann Hematol 2008;87:1003-8.

17. Baron F, Maris MB, Storer BE, et al. High doses of transplanted CD34 + cells are associated with rapid T-cell engraftment and lessened risk of graft rejection, but not more graft-versus-host disease after nonmyeloablative conditioning and unrelated hematopoietic cell transplantation. Leukemia 2005;19:822-8.

18. Mickelson DM, Sproat L, Dean R, et al. Comparison of donor chimerism following myeloablative and nonmyeloablative allogeneic hematopoietic SCT. Bone Marrow Transplant 2011; 46:84-9.

19. Gonzalez-Vicent M, Diaz MA. Higher doses of CD34+ PBPC are associated with a rapid acquisition of full donor chimerism and lower risk of relapse after allogeneic transplantation in pediatric patients with hematological malignancies. J Pediatr Hematol Oncol 2011;33:185-9.

20. Remberger M, Jaksch M, Uzunel M, Mattsson J. Serum levels of cytokines correlate to donor chimerism and acute graft-vs.-host disease after haematopoietic stem cell transplantation. Eur J Haematol 2003;70:384-91.

21. Jillella AP, Shafer D, Klumpp TR, Emmons RV, Mangan KF. Mixed chimerism and graft failure following conditioning with the fludarabine and cyclophosphamide nonablative regimen; conversion to full donor chimerism. Am J Hematol 2007;82:419-26.

22. Armand P, Kim HT, Cutler CS, et al. Prognostic impact of elevated pretransplantation serum ferritin in patients undergoing myeloablative stem cell transplantation. Blood 2007;109:4586-8.

23. Altes A, Remacha AF, Sureda A, et al. Iron overload might increase transplant-related mortality in haematopoietic stem cell transplantation. Bone Marrow Transplant 2002;29:987-9.

24. Kim YR, Kim JS, Cheong JW, Song JW, Min YH. Transfusionassociated iron overload as an adverse risk factor for transplantation outcome in patients undergoing reduced-intensity stem cell transplantation for myeloid malignancies. Acta Haematol 2008;120:182-9. 